\title{
HYPERON POLARIZATION AND MULTIPLE QUARK SCATTERING*
}

\author{
Jerzy SZWED \\ Physics Department \\ Brookhaven National Laboratory \\ Upton, New York 11973 \\ Jagellonian University** \\ Physics Department \\ Kraków, Poland \\ and \\ Romuald WIT \\ Physics Department \\ Jagellonian University \\ Kraków, Poland
}

BNL- -41988

DE89 003573

\begin{abstract}
The quark scattering off external gluonic field, calculated to second order, and the quark recombination model are used to describe both the $\Lambda$ inclusive production cross-section and the $\Lambda$ polarization. The model gives good agreement with experimental data.
\end{abstract}

\footnotetext{
*Presented by J. Szwed at the Internationa! Symposium on High Energy Spin Physics, Minneapolis, Minn., September 1988.

**Permanent address.
}

This manuscript has been authored under contract number DE-AC02-76CH00016 with the U.S. Depar ment of Energy. Accordingly, the U.S. Government retains a non-exclusive, royalty-free license to publish or reproduce the published form of this contribution, or allow others to do so, for U.S. Government purposes. 


\section{INTRODUCTION}

Hyperon polarization has been one of the most discussed problems in spin physics in the last years. Since its surprising measurement ${ }^{1}$ many attempts have been made to explain its strange behaviour. Its increase with the hyperon transverse momentum (see data in Fig. 1) contradicts the naive belief that the dominance of leading, hard scattering mechanism results in vanishing polarization. One lesson which follows from most of the proposed models ${ }^{2-5}$ is that the kinematical range of the hyperon polarization, as studied experimentally, is inbetween the soft and hard regions. This is probably the main reason for the lack of satisfactory, quantitative description of the effect - neither models of soft, multiparticle production, nor perturbative QCD can separately give the correct answer.

In this talk we study the quantitative consequences of the model proposed by one of us 7 years ago. ${ }^{4}$ The idea consists in the polarization of the massive strange quark in the course of multiple scattering off external gluonic field, which approximates the quarkquark and quark-gluon subprocesses. The model reproduced quantitatively all the features observed in proton and meson induced hyperon production. It was left however on the quark level. Here we present quantitative consequences of this polarization mechanism, "hadronized" with the use of the recombination model. ${ }^{6}$ In this way we take into account the intermediate character of the polarization effect: the second order perturbative $Q C D$ calculation is "married" with the soft, multiparticle production model.

\section{MULTIPLE QUARK SCATTERING}

Among many QCD subprocesses which contribute to high energy hadron- hadron collisions the scattering of massive quarks can lead to nonzero polarization. This interference effect appears already in the second order of perturbative calculus. In our approach we approximate the scattering off quarks and gluons by the scattering off external gluonic field, produced during the collision in the interaction region, of the form:

$$
\Phi^{a}(\vec{q})=\frac{4 \pi g}{|\vec{q}|^{2}} I^{a} \quad a=1, \ldots, 8
$$

with $g$ - the quark-gluon coupling constant, $\vec{q}$ - the momentum transfer and $I^{a}-$ an 8 component vector characterizing the external coloured field. In second order perturbation 
calculus the polarization reads:

$$
P_{q}=\frac{2 C \alpha_{s} m_{q}|\vec{k}|}{E^{2}} \frac{\sin ^{3} \frac{\theta}{2} \log \sin \frac{\theta}{2}}{\left(1-\frac{\vec{k}^{2}}{\bar{E}^{2}} \sin ^{2} \frac{\theta}{2}\right) \cos \frac{\theta}{2}} \hat{\nu}
$$

where

$$
C=\frac{d_{a b c} I^{a} I^{b} I^{c}}{I^{a} I^{a}}
$$

$m_{q}, \vec{k}_{i / f}, \mathrm{E}$ and $\theta$ are the mass, initial/final momentum, energy and scattering angle of the quark. The unit vector

$$
\hat{\nu}=\frac{\vec{k}_{i} \times \vec{k}_{f}}{\left|\vec{k}_{i} \times \vec{k}_{f}\right|}
$$

points in the direction perpendicular to the scattering plane. The coupling constant $\alpha_{s}=$ $g^{2} / 4 \pi$ is allowed to run for the transverse momentum greater than $1 \mathrm{GeV} / c$ with $\Lambda_{Q C D}=$ $200 \mathrm{MeV}$. Below this threshold it is kept constant.

The magnitude of the colour constant $\mathrm{C}$ which is given by the strength of the gluonic field can be compared to that of the scattering off another quark. ${ }^{7}$ In that case

$$
C=\frac{1}{16}\left(d_{a b c}\right)^{2}=\frac{5}{6}
$$

One may expect that the effective external field which approximates in our approach the gluonic field of the interaction region is in general stronger than that following from quarkquark scattering.

There is no clear reason to break the calculation at second order. Instead of computing higher order diagrams the expression for $P_{q}$ was compared numerically with the exact solution of the Dirac equation in external field. ${ }^{8}$ Both calculations give approximately the same result which encourages us to use the above expression for $P_{q}$ in our polarization model.

The question of appropriate reference frame where the above approximation can be consistently formulated, was discussed in Ref. 9. Since the scattering off external field transfers only 3-momentum and not energy, one should apply the polarization formula in the frame in which the scattered quark fullfils this condition.

THE RECOMBINATION MODEL

The basic idea of the model follows from the similarity of the distribution of mesons produced in hadronic collisions and the distribution of quarks common to the incoming and 
produced hadrons. ${ }^{10}$ The model states that the quarks of the projectile hadron just "fly through" without significant change of their momenta. Quantitatively this means that the cross-section for e.g. baryon inclusive production (in which we are interested in) reads 6,11

$$
\frac{x}{\sigma} \frac{d \sigma}{d x}=\gamma \int d x_{1} d x_{2} x_{1} q\left(x_{1}\right) x_{2} D\left(x_{2}\right)\left(1-x_{1}-x_{2}\right) R\left(x_{1}, x_{2}, x\right)
$$

where $q\left(x_{1}\right)$ and $D\left(x_{2}\right)$ denote the quark and diquark distributions respectively, $\left(1-x_{1}-x_{2}\right)$ is the phase space factor and the recombination function takes the form

$$
R\left(x_{1}, x_{2}, x\right)=\beta \frac{x_{1} x_{2}}{x^{2}} \delta\left(x_{1}+x_{2}-x\right)
$$

The constants $\beta$ and $\gamma$ are left free. There is no explicit transverse momentum dependence in the model and the above cross-section is usually compared with the experimental data integrated over transverse momentum.

The model is quite succesful in describing the inclusive meson production. ${ }^{12}$ It has been also extended to the inclusive baryon production, ${ }^{11}$ in particular the $\boldsymbol{\Lambda}$ production has been fitted in Ref. 13. In the following we are using the resulting ${ }^{13}$ fixed quark and diquark distributions to calculate the $\boldsymbol{\Lambda}$ polarization.

\section{THE LAMBDA POLARIZATION}

Having introduced two basic ingredients of our model - the multiple quark scattering and the recombination procedure, we are able to calculate the hyperon polarization. The only additional assumption follows from the lack of $p_{T}$ dependence in the recombination model. We assume therefore the strange quark momentum to be parallel to the hyperon momentum.

In this talk we concentrate on the $\Lambda$ hyperon which spin is given in the $\mathrm{SU}(6)$ symmetric case by the strange quark spin. Having performed the integral over $x_{2}$ the $\Lambda$ polarization dependence on Feynman $x$ and transverse momentum $p_{T}$ is given by

$$
P_{\Lambda}\left(x, p_{T}\right)=\frac{\int d x_{1} x_{1} D\left(x_{1}\right)\left(x-x_{1}\right) q\left(x-x_{1}\right) P_{q}\left(x-x_{1}, x p_{T}\right)}{\int d x_{1} x_{1} D\left(x_{1}\right)\left(x-x_{1}\right) q\left(x-x_{1}\right)}
$$

where $D\left(x_{1}\right)$ and $q\left(x-x_{1}\right)$ are now the $(u d)_{S=0}$ diquark and s-quark distributions respectively. The above formula is compared in Fig. 1. with the data on $\Lambda$ production off berylium target at $400 \mathrm{GeV} / \mathrm{c}^{14}$ The solid line corresponds to the model as described in 
the text with the coupling constant $\alpha_{s}$ running for $p_{T} \geq 1 \mathrm{GeV} / \mathrm{c}$, the broken one -with $\alpha_{s}$ fixed. The agreement with the data is quite good.

To estimate the strenghth of the coloured field $\mathrm{C}$ we have to take into account the fact that the model should be used for the directly produced $\Delta$ hyperons which means that the data should be multiplied by a factor of $1.4 .^{14}$ In this case $C=1.9$ as compared to $5 / 6$ suggested by the gluon field resulting from a single quark. If however the scale of the transverse polarization is given not by the quark mass $\left(m_{s}=.5 \mathrm{GeV}\right)$ but by the hadron mass ${ }^{15}$ - the constant $C$ comes out close to 1.

\section{CONCLUSIONS}

We have presented a quantitative model of hyperon polarization. Since the transverse momentum covered by experiments is inbetween soft and hard regions we have "borrowed" from one region the soft recombination model and from the other - the hard scattering off external gluonic field. This we consider as the best we could use in the area where perturbative and nonperturbative effects interfere. We are aware of the fact that the above calculation is a QCD - inspired and compatible model rather than a QCD result. Keeping this in mind we claim good agreement of the model with the data.

The polarization of other hyperons requires additional knowledge concerning the wave functions. One knows that SU(6) symmetry is broken in the structure functions of baryons, one has also to take into account possible diquark polarization. Work along these lines is in progress.

To summarize, the QCD picture where the massive quark scatters off external gluonic field in the interaction region seems to be responsible for the $\boldsymbol{\Lambda}$ polarization at high transverse momentum.

\section{ACKNOWLEDGEMENTS}

This paper was finnished and written up during the visit of one of us (J.S.) to Brookhaven National Laboratory. He would like to thank William Marciano and the Theory Group of BNL for their hospitality. Discussions with Krzysztof Fialkowski are also acknowledged. 

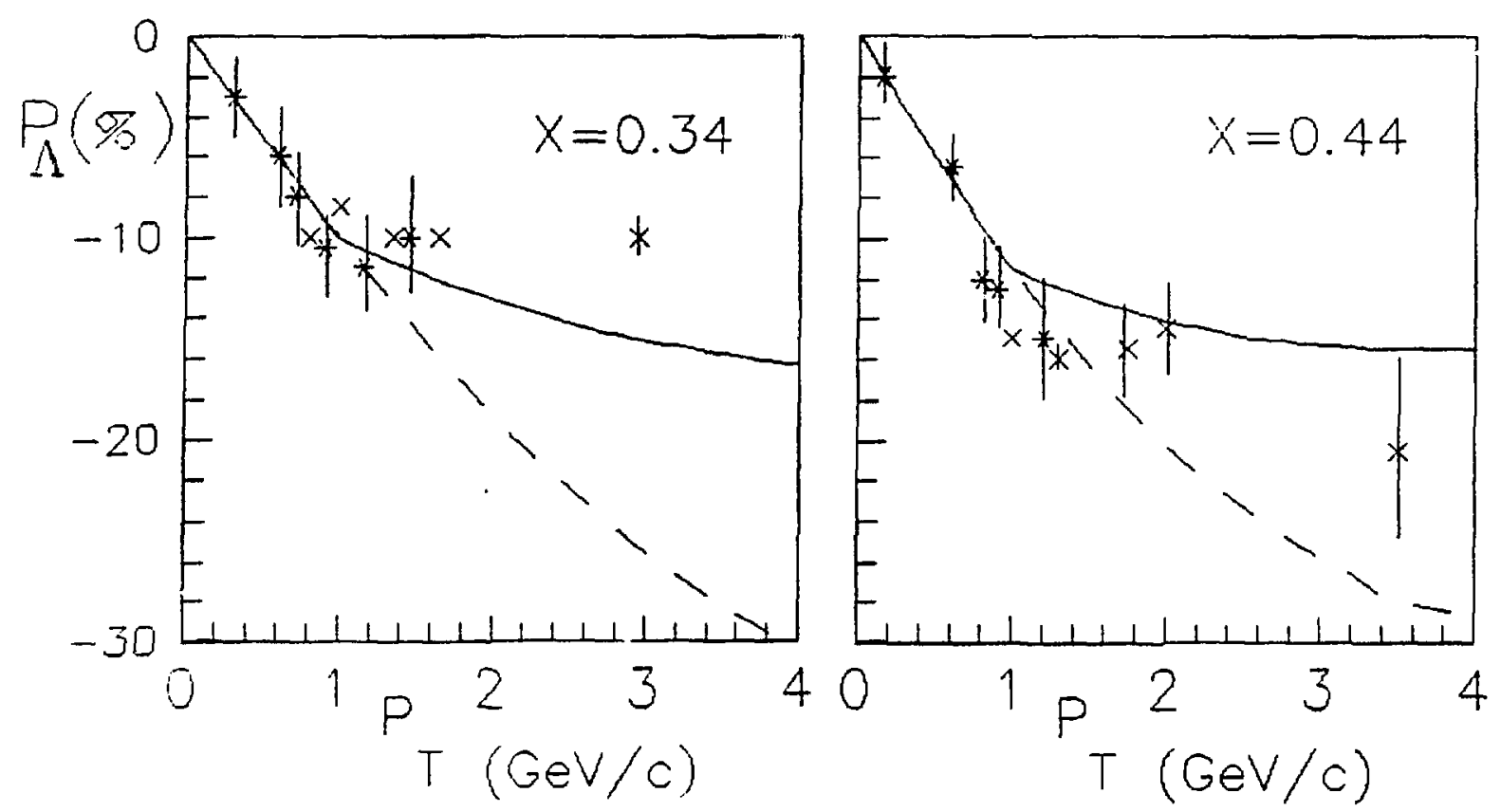

Fig. 1: Fig. 1. The data on $\Lambda$ polarization ${ }^{14}$ compared with the model described in the text.

\section{REFERENCES}

1. G. Bunce et al., Phys. Rev. Lett. 36, 1113 (1976).

2. B. Anderson, G. Gustafson and G. Ingelman, Phys. Lett. 85B, 417 (1979).

3. T. DeGrand and H. Miettinen, Phys. Rev. D24, 2419 (1981)

4. J. Szwed, Phys. Lett. 105B, 403 (1981).

5. P. Cea, P. Chiapetta, J.P. Guillet and G. Nardulli, Phys. Lett. 193B, 361 (1987).

6. K.P. Das and R.C. Hwa, Phys. Lett. 68B, 459 (1977).

7. W.G.D. Dharmaratna, G.R. Goldstein and G.A. Ringland, to be published in Zeit. f. Physik.

8. N.F. Mott and H.S. Masey, Theory of Atomic Collisions, III ed., Oxford.

9. J.M. Gago, R. Vilela Mendes and P. Vaz, Phys. Lett. 183B, 357 (1987).

10. W. Ochs, Nucl. Phys. B118, 97 (1977).

11. J. Ranft, Phys. Rev. D18, 1491 (1978); Acta Phys. Polon. B10, 911 (1979).

12. for review see K. Fiałkowski and W. Kittel, Rep. Progr. Phys. 46, 1283 (1983). 
13. L.G. Pondrom, Phys. Rep., 122C, 57 (1985).

14. for compilation of data see $K$. Heller, Journ. de Phys. C2 46, 121 (1985).

15. A.V. Efremov and O.V. Teryaev, Sov. J. Nucl. Phys. 36, 140 (1982); Yad. Fiz. 39, 1517 (1985); P.G. Ratcliffe, Nucl. Phys. B264, 493 (1986).

\section{DISCLAIMER}

This report was prepared as an account of work sponsored by an agency of the United States Government. Neither the United States Government nor any agency thereof, nor any of their employees, makes any warranty, express or implied, or assumes any legal liability or responsibility for the accuracy, completeness, or usefulness of any information, apparatus, product, or process disclosed, or represents that its use would not infrirge privately owned rights. Reference herein to any specific commercial product, process, or service by trade name, trademark, manufacturer, or otherwise does not necessarily constitute or imply its endorsement, recommendation, or favoring by the United States Government or any agency thereof. The views and opinions of authors expressed herein do not necessarily state or reflect those of the United States Government or any agency thereof. 